\title{
IR Spectra of Crystalline Nucleobases. Combination of Periodic Harmonic Calculations with Anharmonic Corrections Based on Finite Models
}

\section{SUPPORTING INFORMATION}

Krzysztof B. Beć, ${ }^{1,2^{*}}$ Justyna Grabska, ${ }^{1}$ Mirosław A. Czarnecki, ${ }^{2}$ Christian W. Huck, ${ }^{1}$ Marek J. Wójcik, ${ }^{3}$ Takahito Nakajima, ${ }^{4}$ Yukihiro Ozaki ${ }^{5}$

${ }^{1}$ Institute of Analytical Chemistry, University of Innsbruck, A6020 Innsbruck, AUSTRIA

${ }^{2}$ Faculty of Chemistry, University of Wrocław, F. Joliot-Curie 14, 50-383 Wrocław, POLAND

${ }^{3}$ Faculty of Chemistry, Jagiellonian University, Gronostajowa 2, 30-387

Kraków, POLAND

${ }^{4}$ RIKEN Center for Computational Science, 7-1-26, Minatojima-minami-machi, Chuo-ku, Kobe, Hyogo 650-0047, JAPAN

${ }^{5}$ Department of Chemistry, School of Science and Technology, Kwansei Gakuin University, Sanda, Hyogo 669-1337, JAPAN

* Corresponding author: K. B. Beć, email address: krzysztof.bec@uibk.ac.at, phone: +4351250757383 

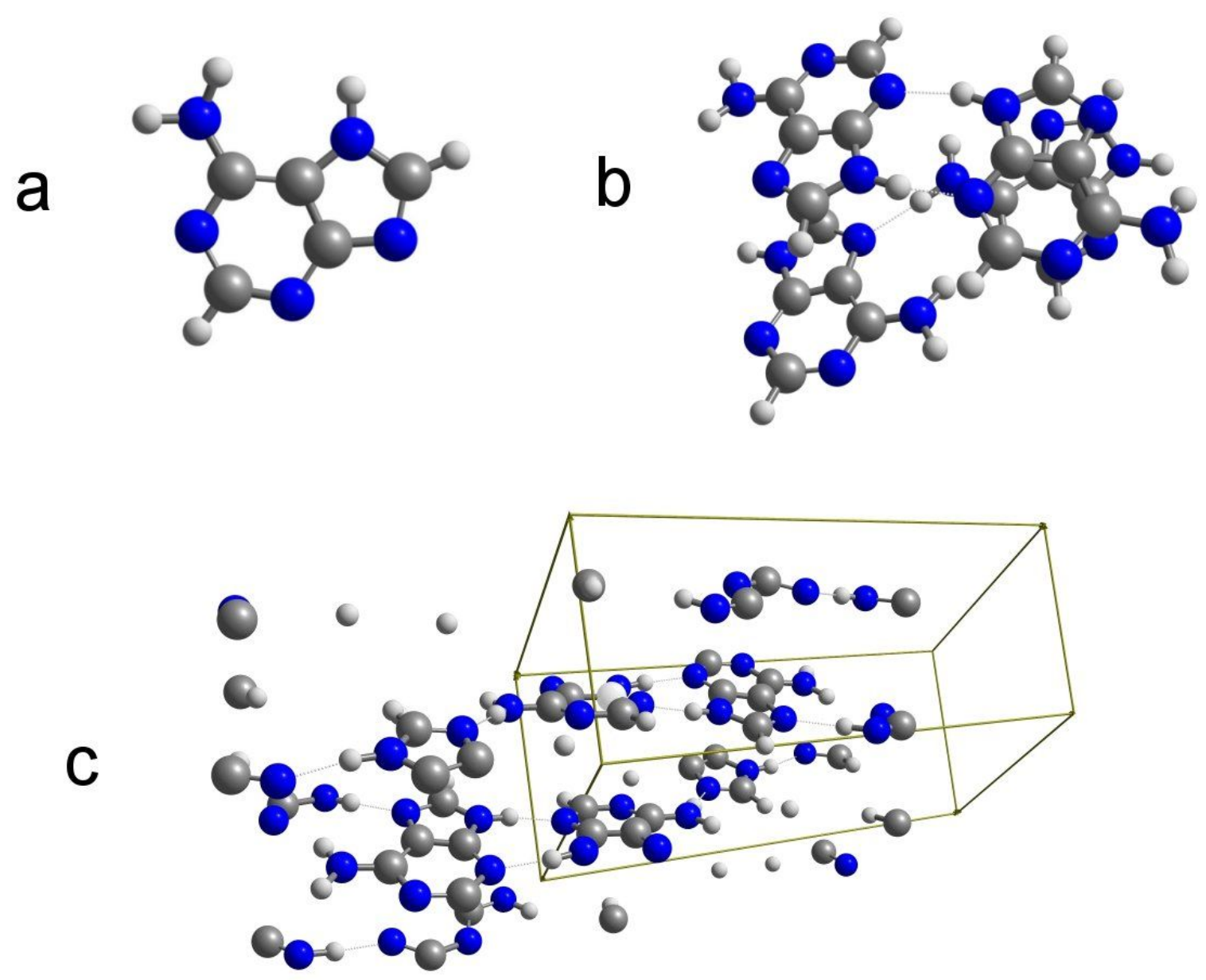

Fig. S1 Molecular Models of adenine used in this study; (a) single molecule; (b) four-molecule cluster extracted from the crystalline structure and optimized in vacuum; (c) three-dimensional periodic boundary system as a near approximation of crystalline structure. 

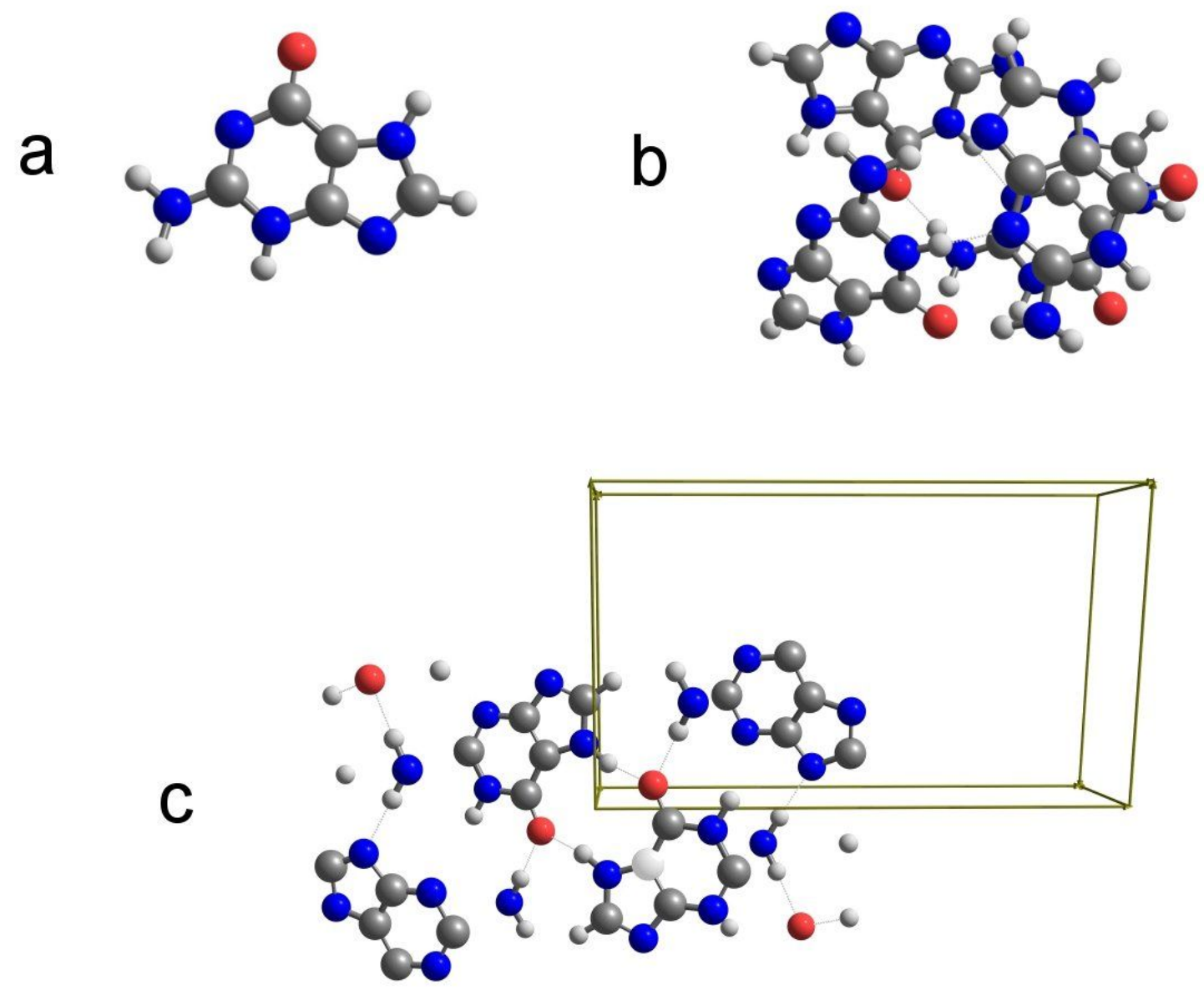

Fig. S2 Molecular Models of guanine used in this study; (a) single molecule; (b) four-molecule cluster extracted from the crystalline structure and optimized in vacuum; (c) three-dimensional periodic boundary system as a near approximation of crystalline structure. 

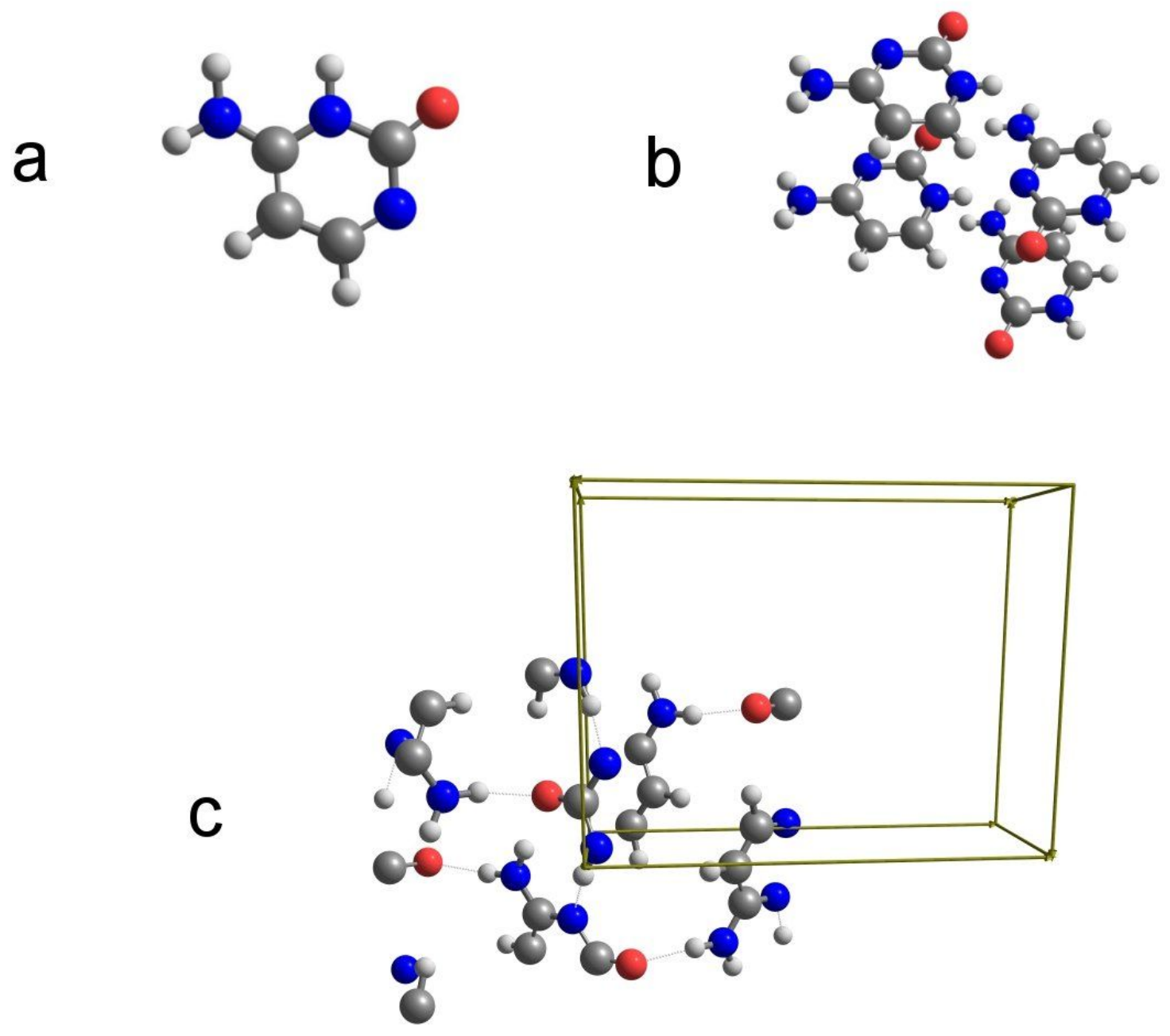

Fig. S3 Molecular Models of cytosine used in this study; (a) single molecule; (b) four-molecule cluster extracted from the crystalline structure and optimized in vacuum; (c) three-dimensional periodic boundary system as a near approximation of crystalline structure. 


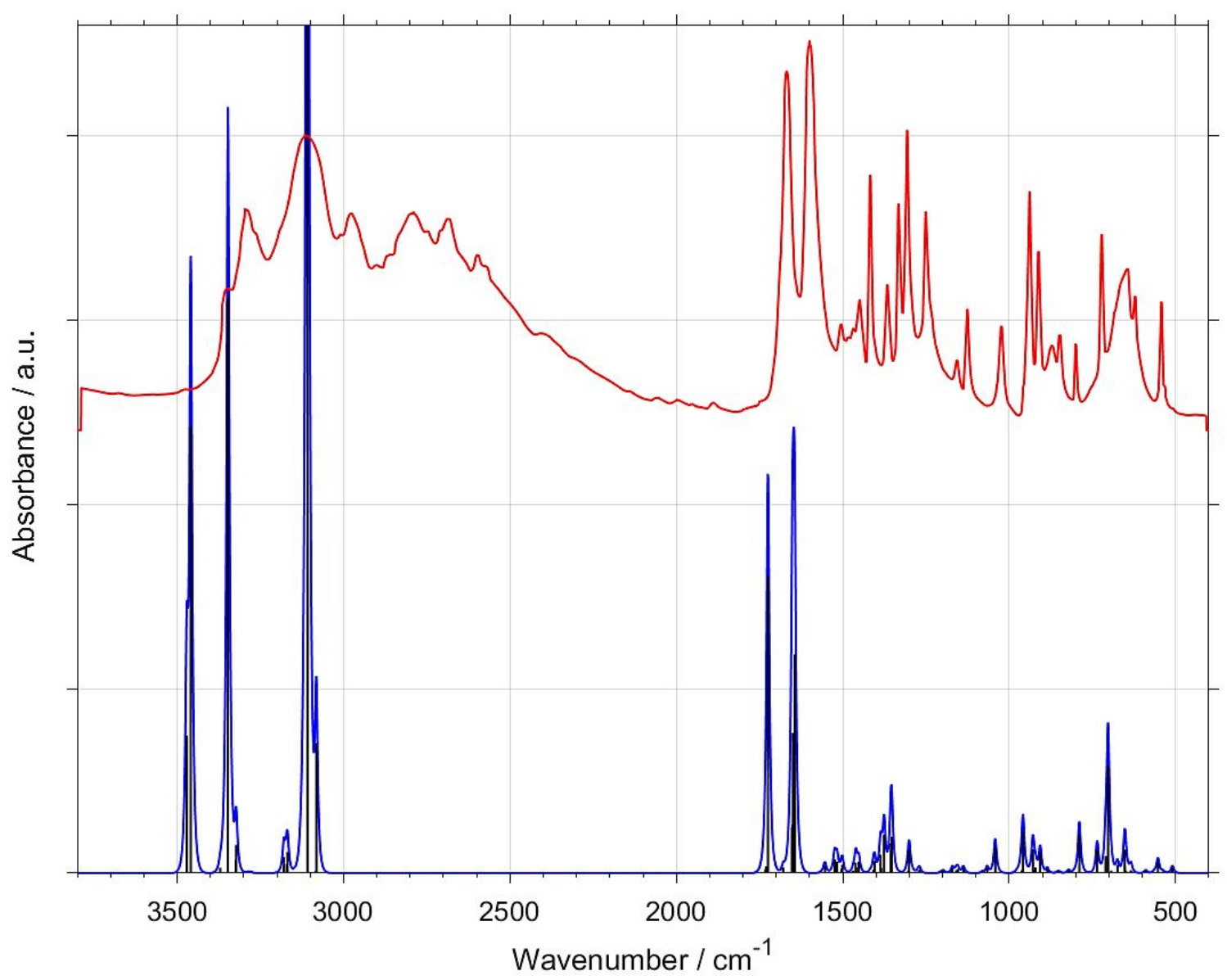

Fig. S4 Experimental and simulated (PBC//B3LYP/Gatti) IR spectra $\left(3800-400 \mathrm{~cm}^{-1}\right)$ of crystalline adenine. The simulated spectrum is shown without wavenumber scaling.

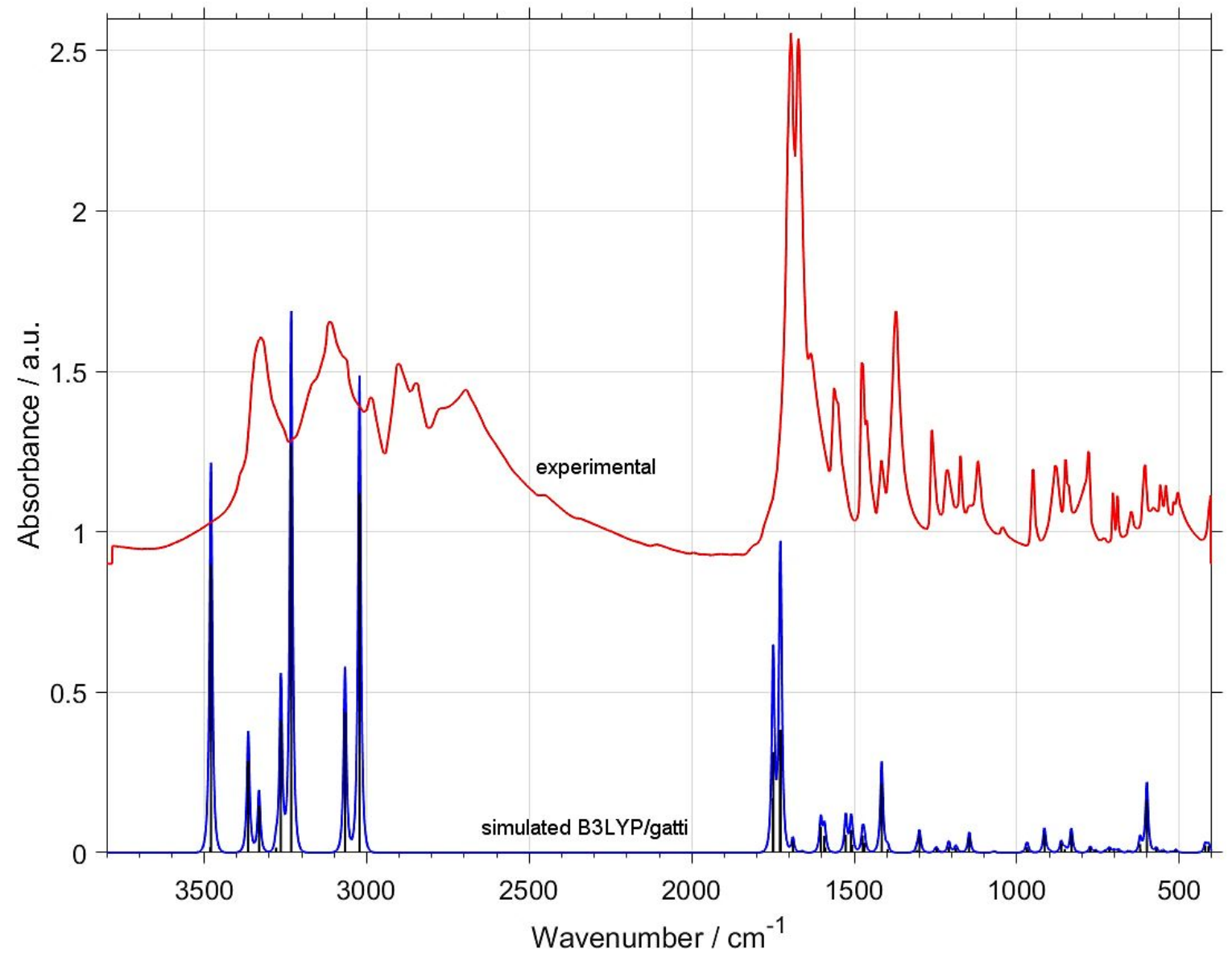

Fig. S5 Experimental and simulated (PBC//B3LYP/Gatti) IR spectra $\left(3800-400 \mathrm{~cm}^{-1}\right)$ of crystalline guanine. The simulated spectrum is shown without wavenumber scaling. 


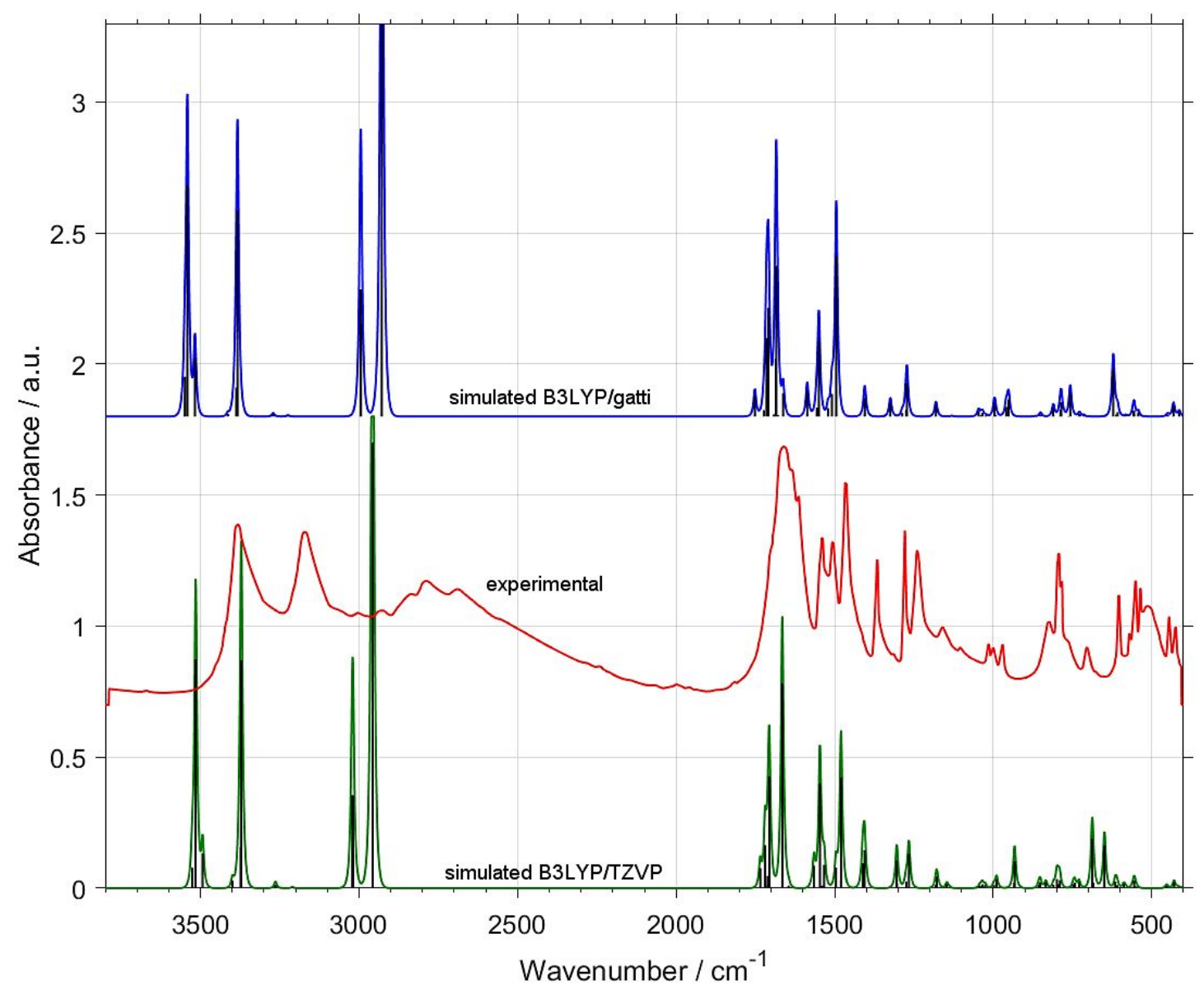

Fig. S6 Experimental and simulated (PBC//B3LYP/Gatti and PBC//B3LYP/TZVP) IR spectra $\left(3800-400 \mathrm{~cm}^{-1}\right)$ of crystalline cytosine. The simulated spectrum is shown without wavenumber scaling.

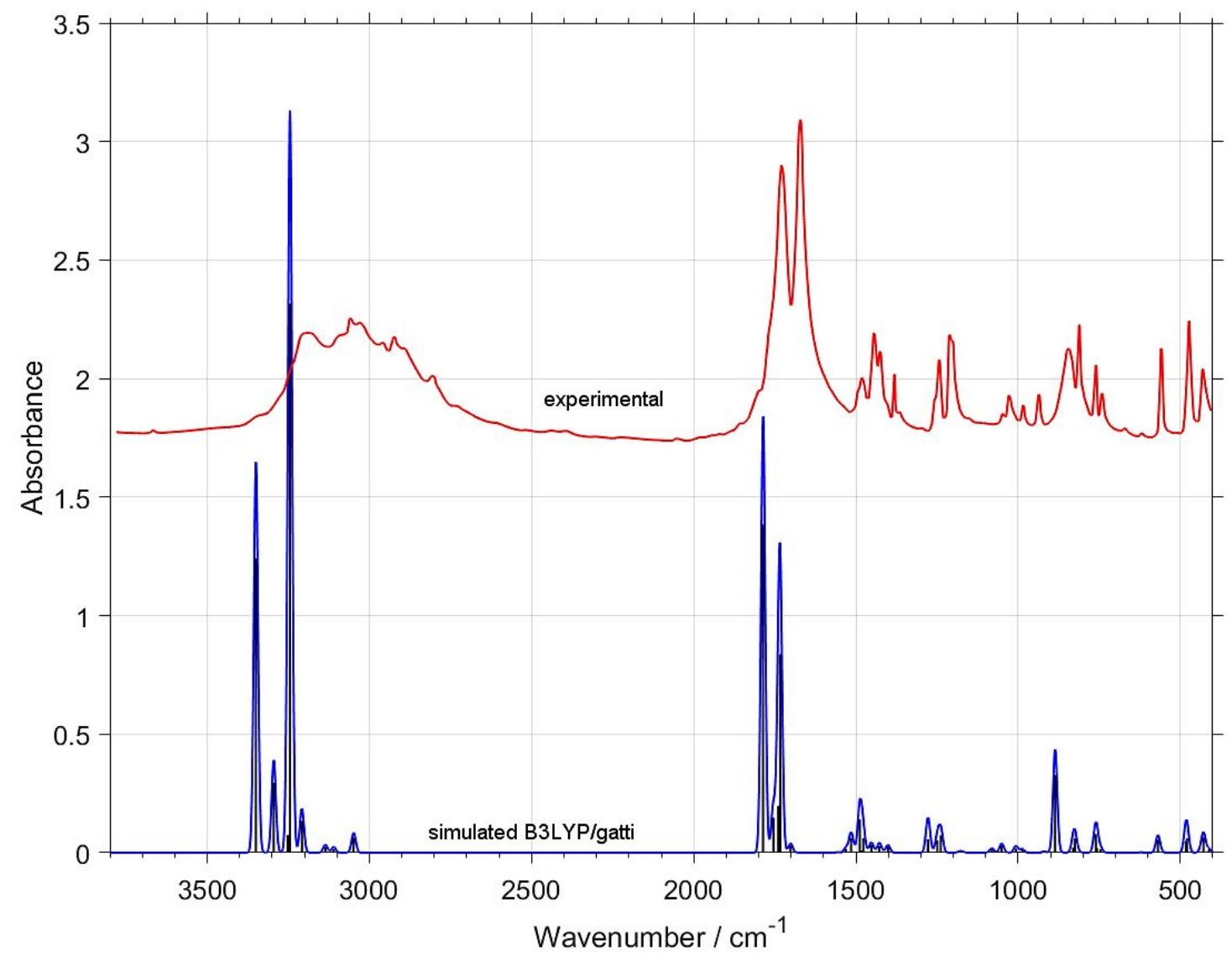

Fig. S7 Experimental and simulated (PBC//B3LYP/Gatti) IR spectra $\left(3800-400 \mathrm{~cm}^{-1}\right)$ of crystalline thymine. The simulated spectrum is shown without wavenumber scaling. 


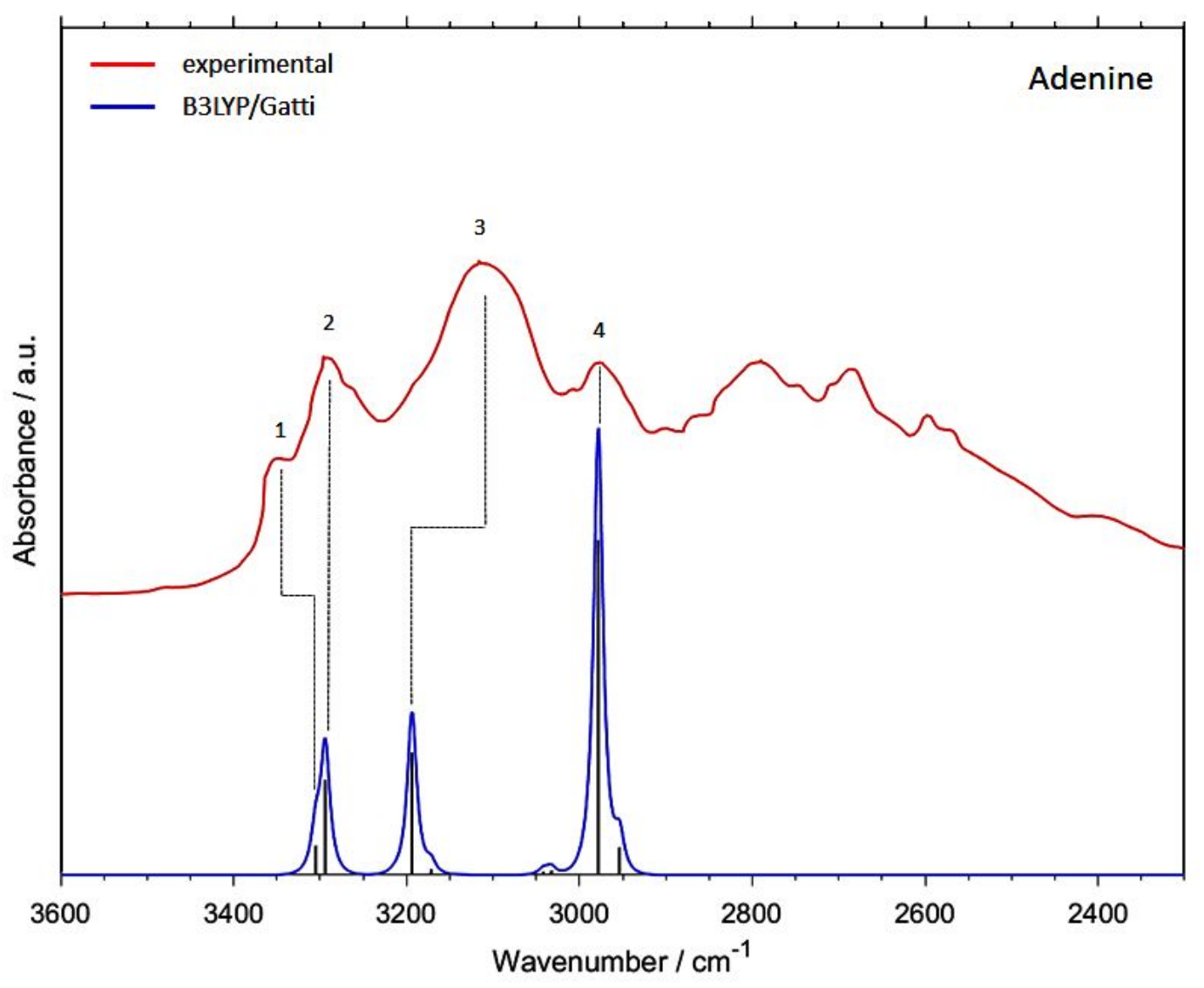

Fig. S8 Experimental and simulated IR spectrum (3600-2300 $\mathrm{cm}^{-1}$ region) of crystalline adenine. Band assignments correspond to those described in Table 1 (main article).

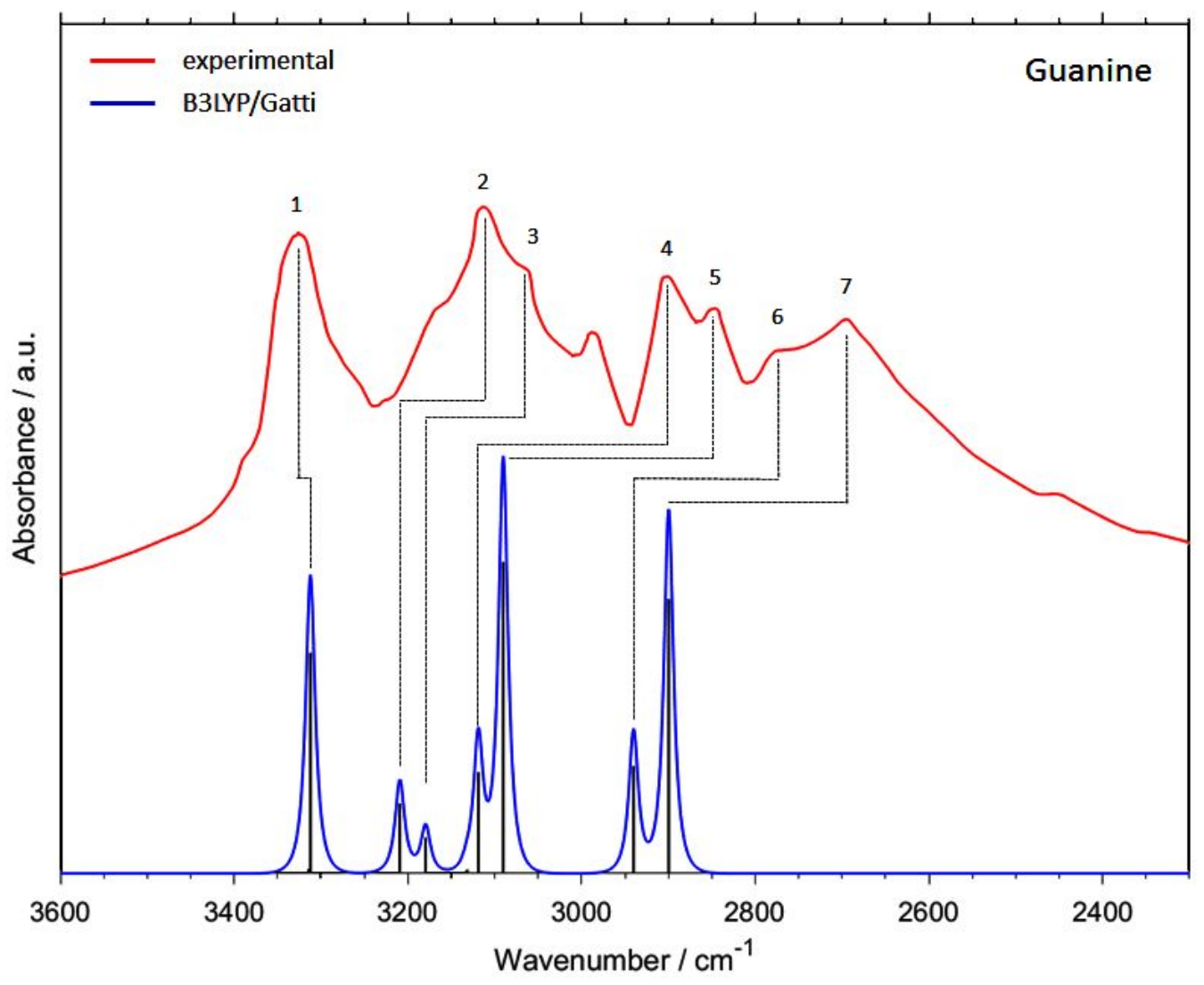

Fig. S9 Experimental and simulated IR spectrum $\left(3600-2300 \mathrm{~cm}^{-1}\right.$ region) of crystalline adenine. Band assignments correspond to those described in Table 1 (main article). 


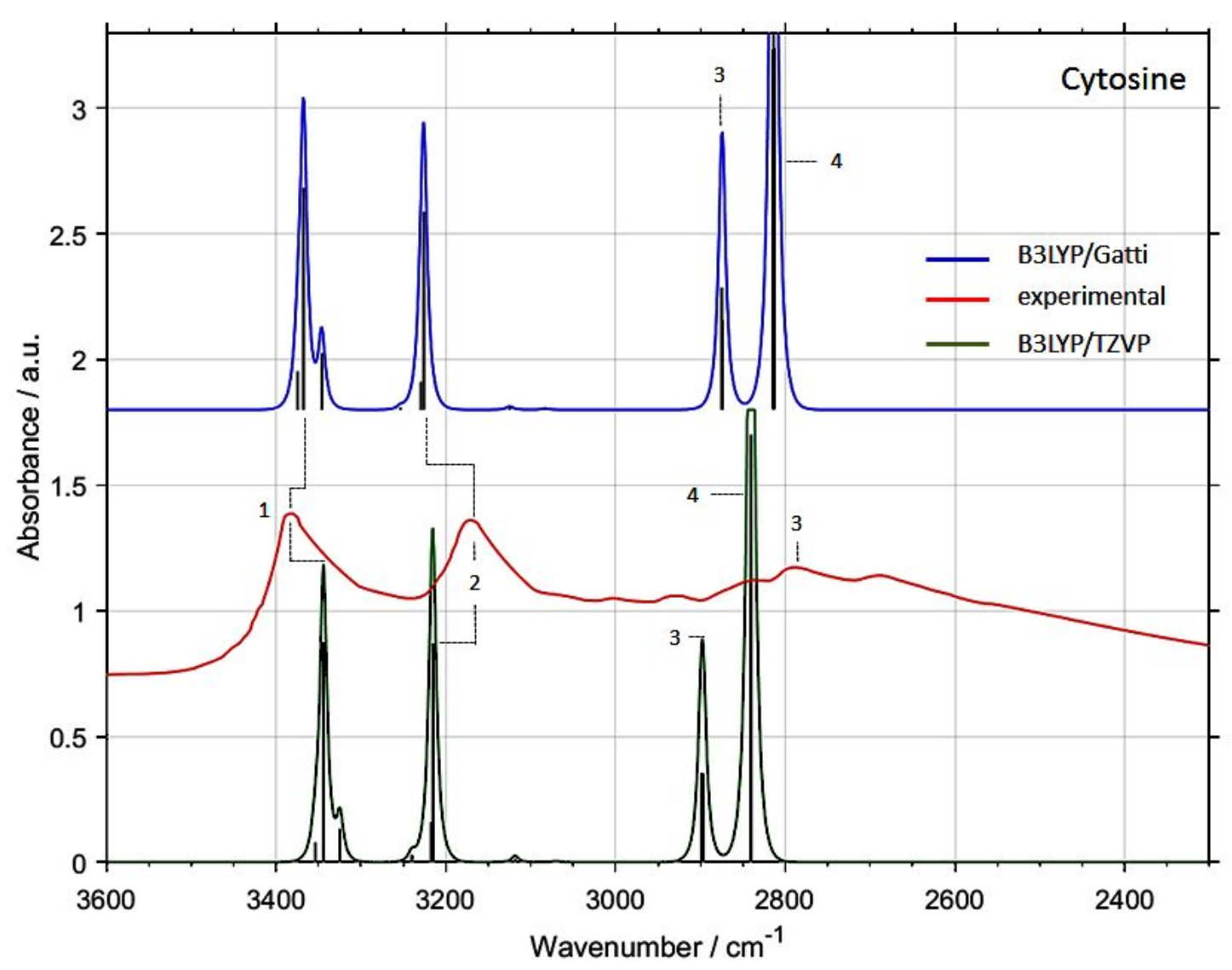

Fig. S10 Experimental and simulated IR spectrum (3600-2300 $\mathrm{cm}^{-1}$ region) of crystalline adenine. Band assignments correspond to those described in Table 1 (main article).

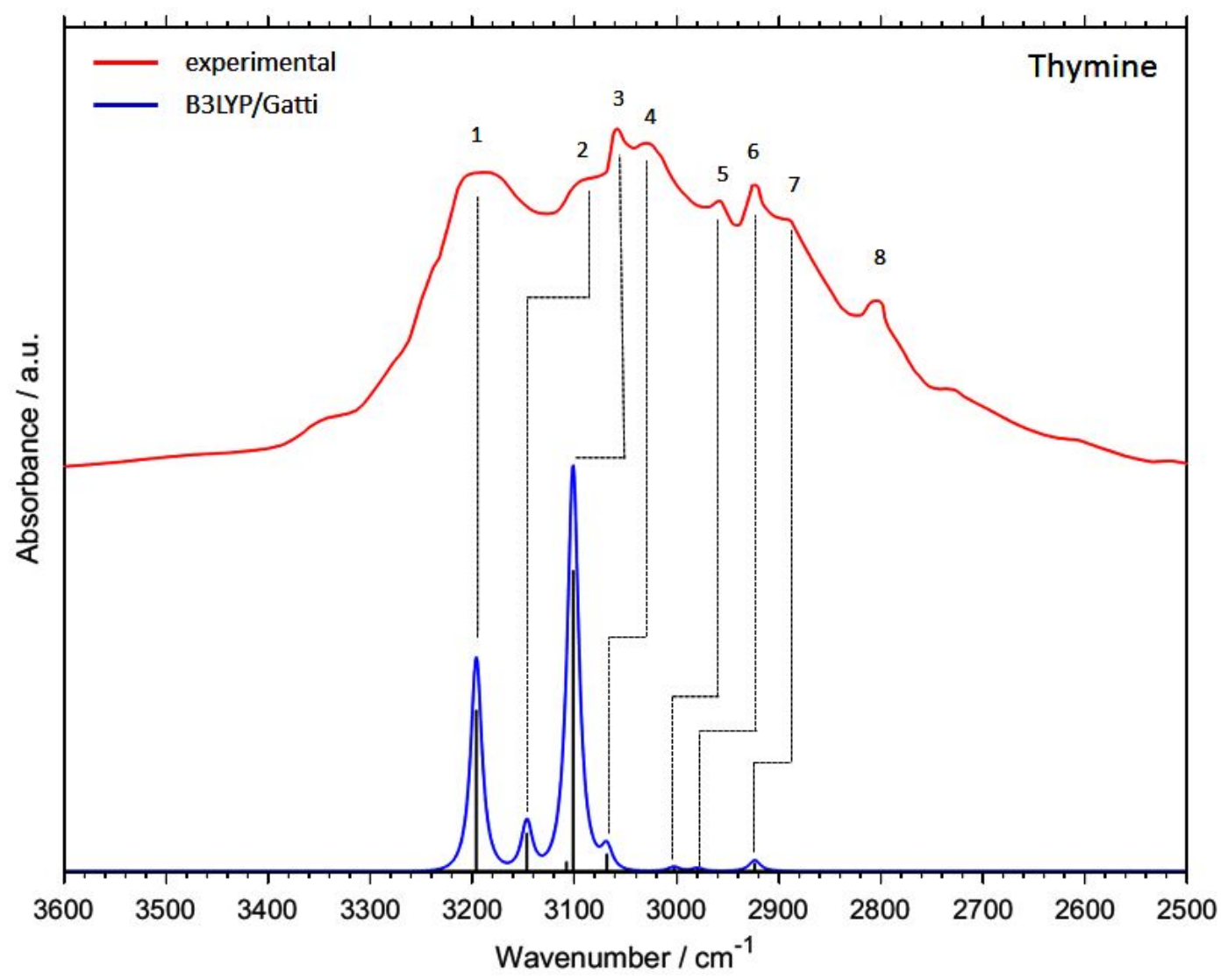

Fig. S11 Experimental and simulated IR spectrum (3600-2300 $\mathrm{cm}^{-1}$ region) of crystalline adenine. Band assignments correspond to those described in Table 1 (main article). 

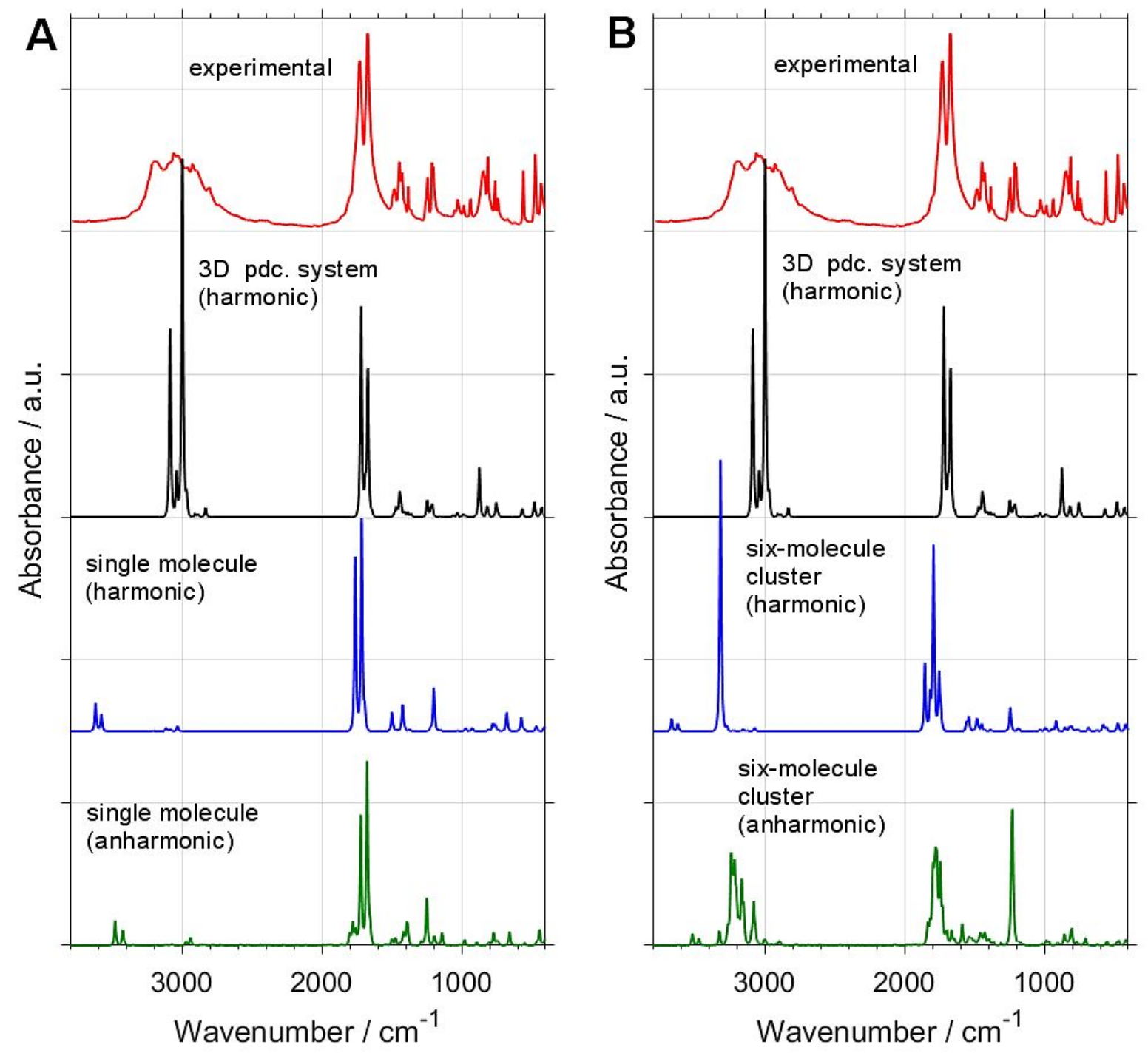

Fig. S12 Comparison of the experimental and simulated IR spectra of thymine $\left(3800-400 \mathrm{~cm}^{-1}\right)$ for single molecule (A) and six-molecule cluster (B). 


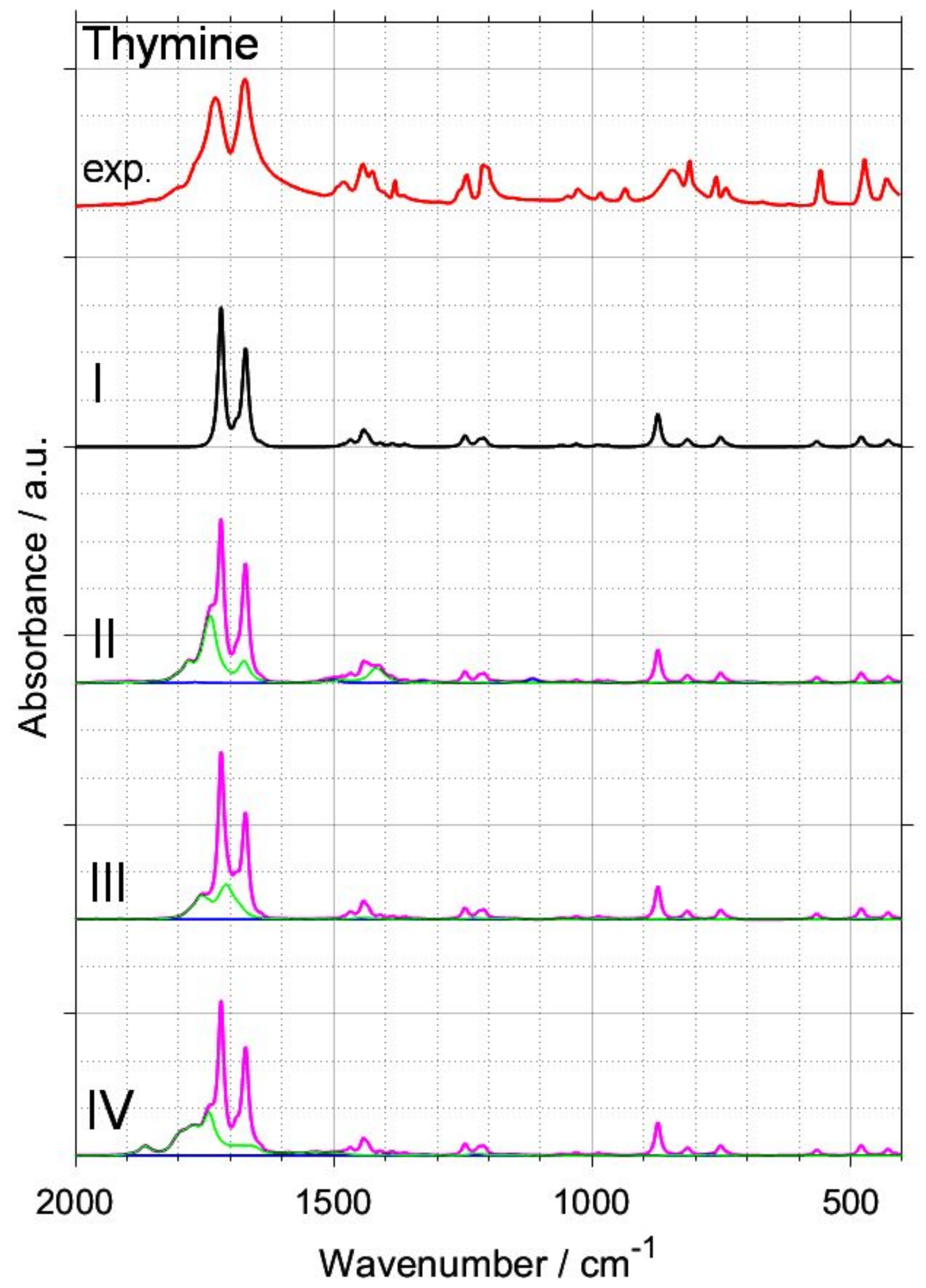

Fig. S13 Experimental (exp.) and simulated IR spectra of thymine in the fingerprint region (2000-400 $\left.\mathrm{cm}^{-1}\right)$. The harmonic spectrum (pdc.//B3LYP/Gatti) in 3D periodic system (I) and after additional anharmonic correction (II-IV); II: DVPT2//B3LYP-GD3BJ/6-31++G(d,p) monomer; III: DVPT2//B3LYP-GD3BJ/6-31G(d,p) dimer. IV: DVPT2//M06-2X/6-31G(d,p) six-molecule cluster. Green line - contribution from binary combinations, blue line- contribution from first overtones. 


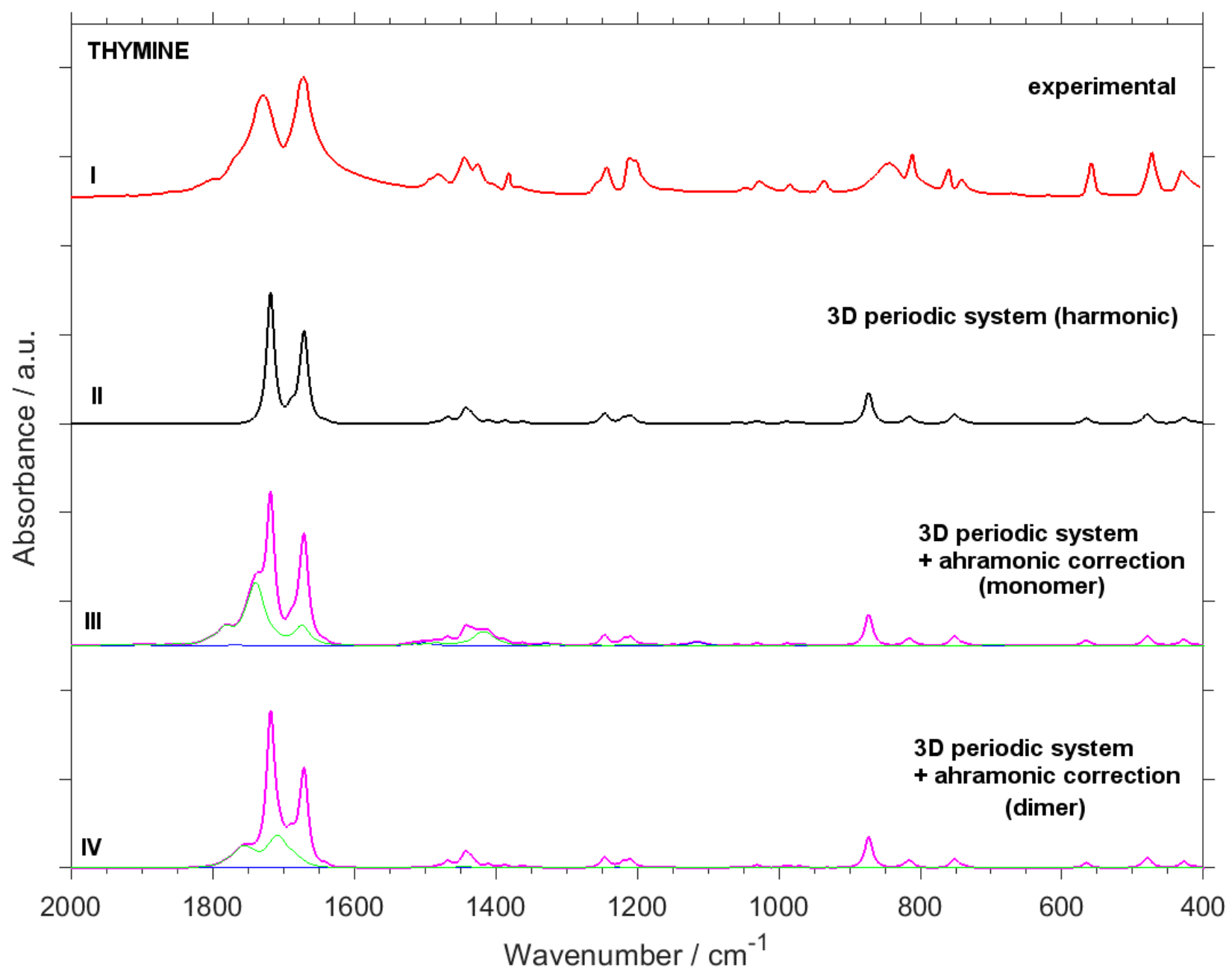

Fig. S14 Experimental and simulated IR spectra of thymine in the fingerprint region $\left(2000-400 \mathrm{~cm}^{-1}\right)$. The harmonic spectrum (pdc.//B3LYP/Gatti) in 3D periodic system (I) and after additional anharmonic correction (II-III); II: DVPT2//B3LYP-GD3BJ/6-31++G(d,p) monomer; III: DVPT2//B3LYP-GD3BJ/6-31G(d,p) dimer. Green line - contribution from binary combinations, blue line- contribution from first overtones. 


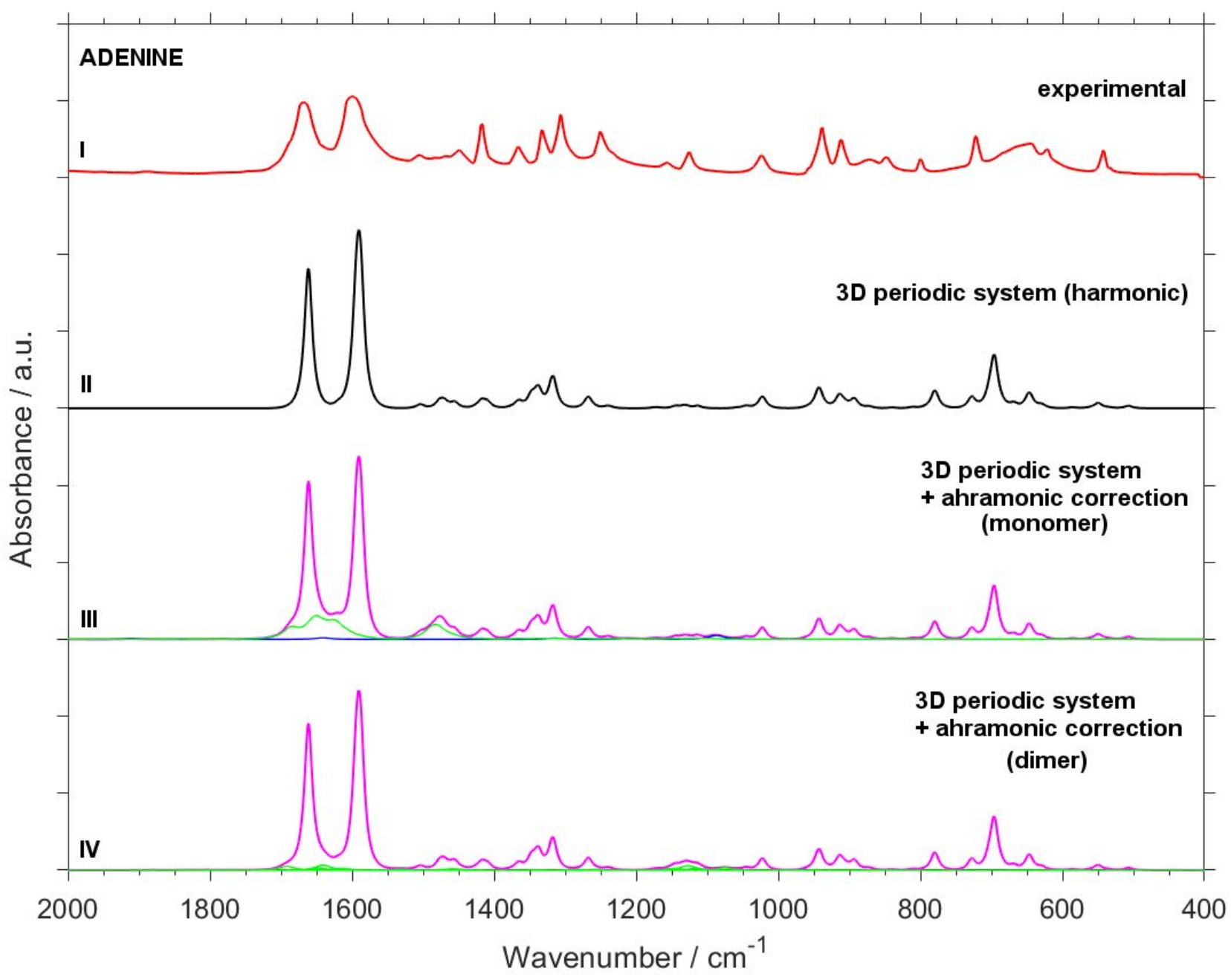

Fig. S15 Experimental and simulated IR spectra of adenine in the fingerprint region $\left(2000-400 \mathrm{~cm}^{-1}\right)$. The harmonic spectrum (pdc.//B3LYP/Gatti) in 3D periodic system (I) and after additional anharmonic correction (II-III); II: DVPT2//B3LYP-GD3BJ/6-31++G(d,p) monomer; III: DVPT2//B3LYP-GD3BJ/6-31G(d,p) dimer. Green line - contribution from binary combinations, blue line- contribution from first overtones. 


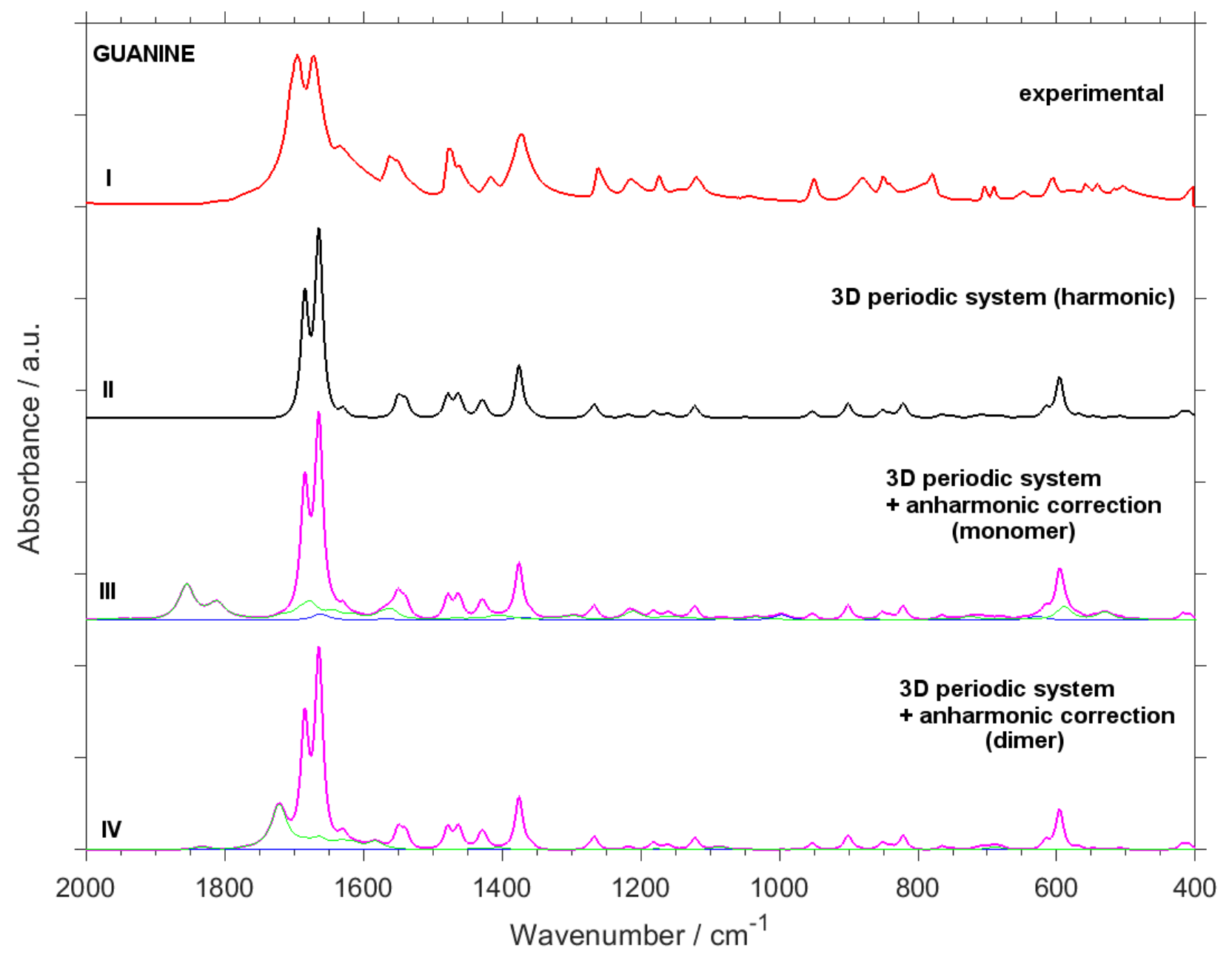

Fig. S16 Experimental and simulated IR spectra of guanine in the fingerprint region $\left(2000-400 \mathrm{~cm}^{-1}\right)$. The harmonic spectrum (pdc.//B3LYP/Gatti) in 3D periodic system (I) and after additional anharmonic correction (II-III); II: DVPT2//B3LYP-GD3BJ/6-31++G(d,p) monomer; III: DVPT2//B3LYP-GD3BJ/6-31G(d,p) dimer. Green line - contribution from binary combinations, blue line- contribution from first overtones. 


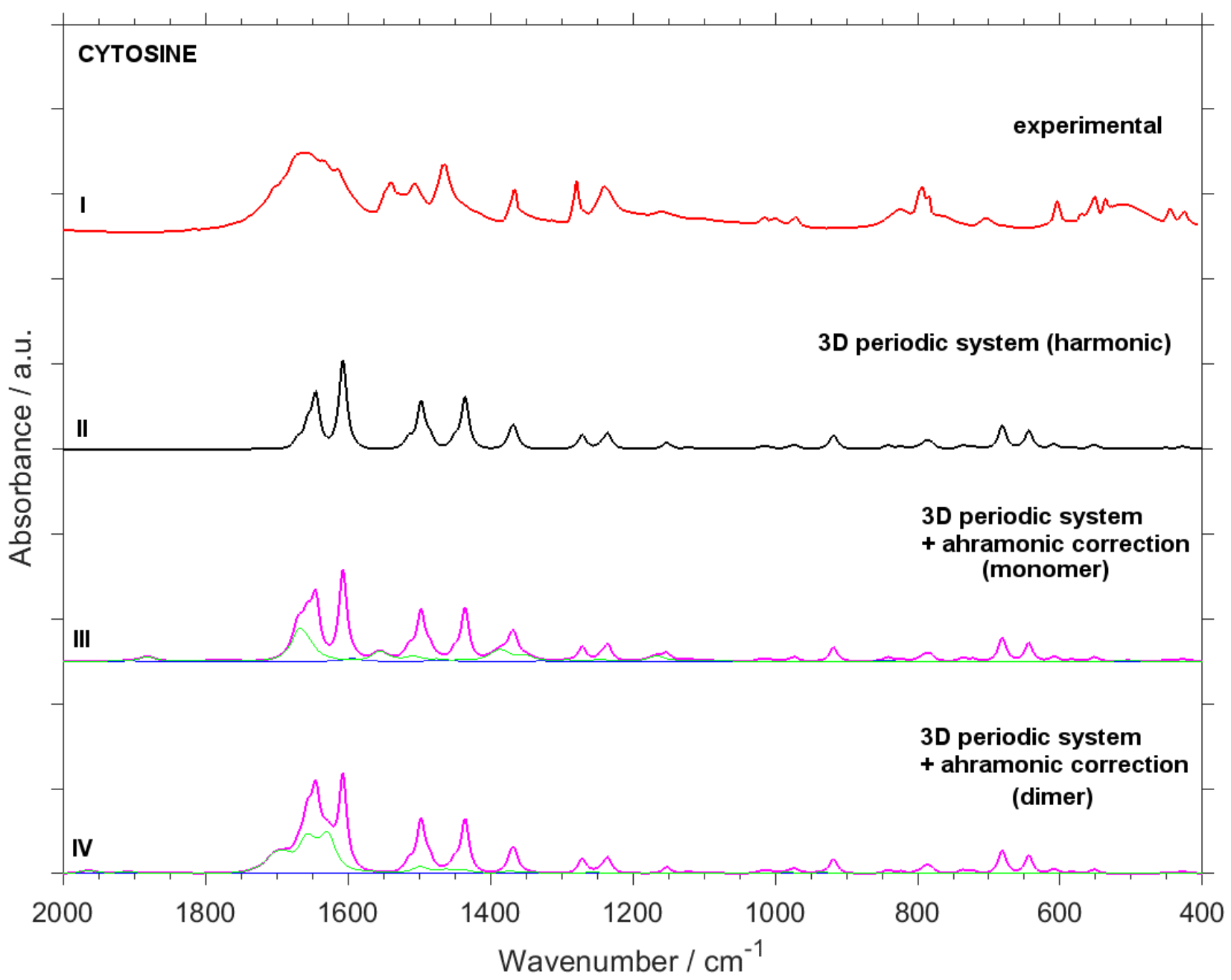

Fig. S17 Experimental and simulated IR spectra of cytosine in the fingerprint region $\left(2000-400 \mathrm{~cm}^{-1}\right)$. The harmonic spectrum (pdc.//B3LYP/Gatti) in 3D periodic system (I) and after additional anharmonic correction (II-III); II: DVPT2//B3LYP-GD3BJ/6-31++G(d,p) monomer; III: DVPT2//B3LYP-GD3BJ/6-31G(d,p) dimer. Green line - contribution from binary combinations, blue line- contribution from first overtones. 

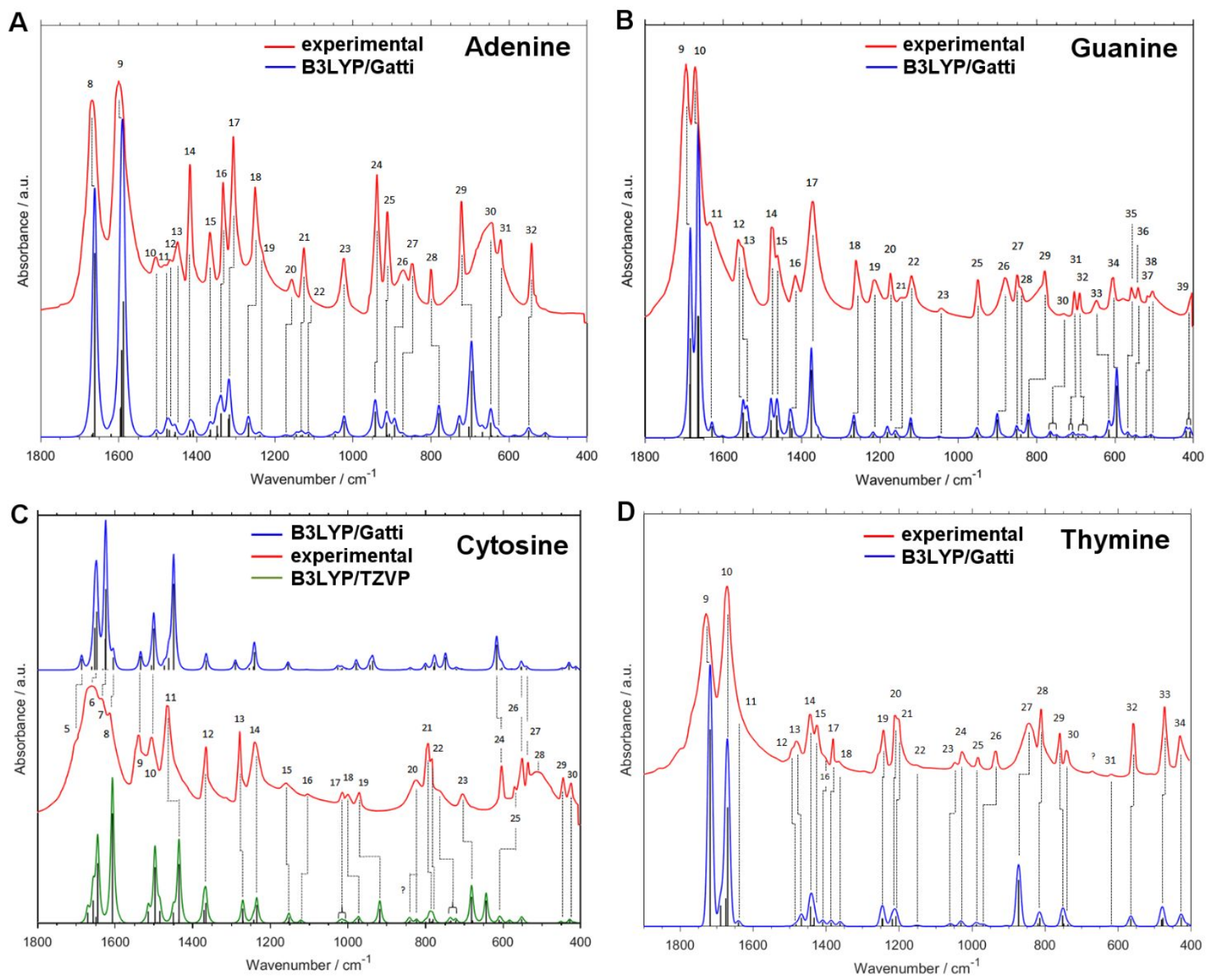

Fig. S18 Experimental and simulated IR spectra of crystalline adenine (A), guanine (B), cytosine (C) and thymine (D) in the fingerprint region (1800-400 cm-1). Band numbers correspond to those in Table 1-4. 


\section{Optimized structures}

Adenine monomer B3LYP/6-31++G(d,p)

$\begin{array}{lrrr}\text { N } & -1.950722000 & 0.523330000 & 0.001522000 \\ \mathrm{C} & -1.300958000 & 1.700168000 & 0.001243000 \\ \mathrm{C} & -1.228400000 & -0.611981000 & -0.001427000 \\ \mathrm{~N} & -1.875534000 & -1.800961000 & -0.012705000 \\ \mathrm{C} & 0.179187000 & -0.519702000 & -0.001596000 \\ \mathrm{~N} & 1.184942000 & -1.474608000 & 0.000283000 \\ \mathrm{C} & 0.709756000 & 0.776100000 & -0.001051000 \\ \mathrm{~N} & 0.018415000 & 1.923029000 & -0.000325000 \\ \mathrm{C} & 2.295764000 & -0.774246000 & 0.001227000 \\ \mathrm{~N} & 2.076215000 & 0.590835000 & 0.000278000 \\ \mathrm{H} & -1.934568000 & 2.582768000 & 0.003151000 \\ \mathrm{H} & -1.354500000 & -2.661330000 & 0.039551000 \\ \mathrm{H} & -2.881663000 & -1.810168000 & 0.040043000 \\ \mathrm{H} & 3.294554000 & -1.187899000 & 0.002759000 \\ \mathrm{H} & 2.770866000 & 1.323220000 & 0.000751000\end{array}$

Adenine dimer B3LYP/6-31++G(d,p)

$\begin{array}{lccc}\text { N } & 3.930077000 & 1.683632000 & 0.000043000 \\ \mathrm{C} & 2.619345000 & 1.955481000 & -0.000002000 \\ \mathrm{C} & 4.321850000 & 0.392677000 & 0.000047000 \\ \mathrm{~N} & 5.643849000 & 0.113697000 & 0.000087000 \\ \mathrm{C} & 3.338774000 & -0.617458000 & 0.000006000 \\ \mathrm{~N} & 3.413500000 & -1.999137000 & 0.000006000 \\ \mathrm{C} & 2.004026000 & -0.191550000 & -0.000039000 \\ \mathrm{~N} & 1.593765000 & 1.093229000 & -0.000044000 \\ \mathrm{C} & 2.152665000 & -2.383351000 & -0.000054000 \\ \mathrm{~N} & 1.253575000 & -1.339644000 & -0.000073000 \\ \mathrm{H} & 2.357308000 & 3.010177000 & -0.000004000 \\ \mathrm{H} & 5.958450000 & -0.843376000 & 0.000131000 \\ \mathrm{H} & 6.307762000 & 0.871559000 & 0.000155000 \\ \mathrm{H} & 1.820855000 & -3.412306000 & -0.000076000 \\ \mathrm{H} & 0.213812000 & -1.385407000 & -0.000113000 \\ \mathrm{~N} & -3.930077000 & -1.683632000 & 0.000046000 \\ \mathrm{C} & -2.619345000 & -1.955481000 & 0.000002000 \\ \mathrm{C} & -4.321850000 & -0.392677000 & 0.000047000 \\ \mathrm{~N} & -5.643849000 & -0.113697000 & 0.000077000 \\ \mathrm{C} & -3.338774000 & 0.617458000 & 0.000005000 \\ \mathrm{~N} & -3.413500000 & 1.999137000 & -0.000001000 \\ \mathrm{C} & -2.004026000 & 0.191550000 & -0.000037000 \\ \mathrm{~N} & -1.593765000 & -1.093229000 & -0.000041000 \\ \mathrm{C} & -2.152665000 & 2.383351000 & -0.000048000 \\ \mathrm{~N} & -1.253575000 & 1.339644000 & -0.000070000 \\ \mathrm{H} & -2.357307000 & -3.010177000 & 0.000002000 \\ \mathrm{H} & -5.958450000 & 0.843376000 & 0.000139000 \\ \mathrm{H} & -6.307761000 & -0.871559000 & 0.000166000 \\ \mathrm{H} & -1.820855000 & 3.412306000 & -0.000067000 \\ \mathrm{H} & -0.213811000 & 1.385408000 & -0.000109000\end{array}$


(6)

$-3.223785000$

2.767105000

1.451384000

$-4.458514000$

3.018816000

$-4.886376000$

4.003877000

1.343717000

4.206558000

1.077085000

$-3.238034000$

1.105804000

0.954079000

$-2.297726000$

1.990931000

0.227431000

$-2.458126000$

3.054139000

$-1.874103000$

$-1.612946000$

3.687226000

$-1.001956000$

$-1.510731000$

4.637522000

$-0.731684000$

1.459286000

3.068653000

$-2.152488000$

1.245754000

2.712611000

$-0.211861000$

1.015893000

2.931507000

0.766895000

3.350769000

3.762042000

2.020575000

2.423289000

5.597319000

3.176193000

0.107858000

5.826005000

4.131346000

$-0.062321000$

4.290368000

2.863616000

0.168603000

3.616926000

$-3.181021000$

$-0.249950000$

2.700676000

$-4.111563000$

0.354841000

3.881169000

1.621426000

0.058263000

4.580486000

0.508564000

$-0.772353000$

6.302222000

2.594632000

$-4.619929000$

$-0.505356000$

$-1.208880000$

$-0.489636000$

0.961828000

$-4.362267000$

0.805984000

1.873774000

1.817171000

089325000

$-5.198179000$

1.383157000

0.855699000

0.509687000

$-3.147128000$

$-2.001726000$

1.469080000

1.721991000

$-0.828883000$

1.370650000

$-2.316791000$

0.925585000

1.611327000

$-0.852534000$

0.274506000

2.078451000

$-0.059473000$

1.917952000

2.121847000

2.054901000

$-0.675157000$

$-0.778698000$

2.000255000

$-3.543111000$

$-1.241661000$

1.245622000

1.158547000

$-3.664511000$

$-2.318435000$

$-2.234361000$

1.535361000

$-2.075639000$

0.499000000

1.728408000

1.546134000

2.302350000

$-0.541623000$

2.060348000

3.441826000

$-0.060482000$

$-1.203028000$

0.082999000

$-1.614253000$

$-1.047246000$

1.435955000

$-1.446817000$

$-0.177982000$

1.897937000

$-1.125936000$

$-2.567103000$

$-0.108001000$

$-1.872777000$

$-4.992764000$

$-1.094736000$

$-1.985869000$

1.388955000

$-4.108621000$

0.334713000

$-0.460099000$

$-3.085894000$

1.287966000

$-0.981737000$

$-3.836738000$

0.857798000

0.855058000

$-3.060558000$

1.004960000

2.359486000

0.142743000

$-1.383522000$

1.450319000

$-0.361158000$

$-1.495061000$

2.498705000

1.409179000

$-0.876020000$

3.634532000

$-0.342903000$

$-1.562177000$

3.811805000

$-1.345964000$

$-1.923197000$

$-0.272167000$

$-0.889589000$

$-1.562345000$

$7-2.077169000$

$-2.430273000$

$-1.960555000$

$-4.290781000$

$-2.246217000$ 


$\begin{array}{llll}1 & -4.529633000 & -2.748395000 & -2.147504000 \\ 6 & -0.794061000 & -2.109480000 & -1.751531000 \\ 1 & -0.096136000 & -2.940885000 & -1.717113000 \\ 6 & -2.993539000 & -1.442283000 & -2.024057000 \\ 1 & 3.062806000 & -4.982101000 & -0.482715000\end{array}$

\section{Cytosine monomer B3LYP/6-31++G(d,p)}

$\begin{array}{cccc}\mathrm{N} & 0.044294000 & -0.993956000 & -0.000800000 \\ \mathrm{~N} & 2.328993000 & -0.916690000 & -0.036523000 \\ \mathrm{~N} & -1.289740000 & 0.977883000 & 0.002402000 \\ \mathrm{C} & 1.147760000 & -0.249616000 & -0.004262000 \\ \mathrm{C} & 1.088462000 & 1.155375000 & -0.001701000 \\ \mathrm{C} & -1.102296000 & -0.335386000 & -0.000014000 \\ \mathrm{C} & -0.168931000 & 1.705189000 & 0.002358000 \\ \mathrm{H} & 1.979243000 & 1.764069000 & -0.007247000 \\ \mathrm{H} & -0.299267000 & 2.780766000 & 0.005295000 \\ \mathrm{H} & 3.187164000 & -0.432503000 & 0.149234000 \\ \mathrm{H} & 2.300317000 & -1.911717000 & 0.103237000 \\ \mathrm{O} & -2.198436000 & -1.102417000 & 0.001516000 \\ \mathrm{H} & -2.954775000 & -0.505305000 & 0.003515000\end{array}$

Cytosine dimer B3LYP/6-31++G(d,p)

$\begin{array}{cccc}\text { O } & -0.968674000 & -1.478886000 & 0.013516000 \\ \text { N } & -3.222110000 & -1.067156000 & 0.005791000 \\ \text { N } & -5.463415000 & -0.611576000 & -0.031235000 \\ \text { C } & -4.182766000 & -0.151959000 & -0.004793000 \\ \text { C } & -3.931391000 & 1.258314000 & -0.011041000 \\ \text { C } & -1.920162000 & -0.673693000 & 0.005887000 \\ \text { C } & -2.617998000 & 1.629064000 & -0.006652000 \\ \text { H } & -4.729159000 & 1.989431000 & -0.024166000 \\ \text { H } & -2.291485000 & 2.663416000 & -0.009943000 \\ \text { H } & -6.242410000 & 0.009036000 & 0.112781000 \\ \text { H } & -5.604531000 & -1.605031000 & 0.075120000 \\ \text { N } & -1.639497000 & 0.698987000 & 0.001388000 \\ \text { H } & -0.635316000 & 0.989908000 & 0.005679000 \\ \text { O } & 0.968681000 & 1.478893000 & 0.013506000 \\ \text { N } & 3.222115000 & 1.067155000 & 0.005849000 \\ \text { N } & 5.463419000 & 0.611568000 & -0.031118000 \\ \text { C } & 4.182767000 & 0.151955000 & -0.004737000 \\ \text { C } & 3.931384000 & -1.258317000 & -0.011044000 \\ \text { C } & 1.920166000 & 0.673696000 & 0.005886000 \\ \text { C } & 2.617989000 & -1.629062000 & -0.006713000 \\ \text { H } & 4.729149000 & -1.989438000 & -0.024166000 \\ \text { H } & 2.291470000 & -2.663412000 & -0.010048000 \\ \text { H } & 6.242402000 & -0.009052000 & 0.112933000 \\ \text { H } & 5.604531000 & 1.605016000 & 0.075297000 \\ \text { N } & 1.639495000 & -0.698981000 & 0.001319000 \\ \text { H } & 0.635310000 & -0.989890000 & 0.005609000\end{array}$




\begin{tabular}{rrrr}
1 & 4.782103000 & 0.142405000 & -1.895039000 \\
1 & 1.862550000 & -3.500320000 & -2.243786000 \\
6 & 3.592757000 & 1.217104000 & -0.597903000 \\
8 & 3.497272000 & 2.152651000 & -1.390325000 \\
6 & -0.651774000 & 3.995706000 & 0.050579000 \\
6 & -0.026985000 & 3.569465000 & -1.161375000 \\
6 & 0.380778000 & 2.267561000 & -1.203643000 \\
1 & 0.157908000 & 4.245110000 & -1.985893000 \\
1 & 0.906524000 & 1.820049000 & -2.038196000 \\
1 & -1.388044000 & 5.572514000 & 1.087617000 \\
1 & -0.801798000 & 5.977186000 & -0.483124000 \\
7 & -1.125651000 & 5.271734000 & 0.159725000 \\
6 & 1.235797000 & -1.802907000 & -1.262391000 \\
8 & 1.593479000 & -1.055154000 & -2.173622000 \\
6 & -5.039761000 & -0.487784000 & -0.25885600 \\
6 & -4.326672000 & 0.694670000 & -0.650878000 \\
6 & -2.980450000 & 0.561043000 & -0.799990000 \\
1 & -4.820655000 & 1.644012000 & -0.810269000 \\
1 & -2.329632000 & 1.380623000 & -1.078402000 \\
1 & -6.843709000 & -1.242454000 & 0.290889000 \\
1 & -6.865637000 & 0.454493000 & -0.051465000 \\
7 & 0.141388000 & 1.458398000 & -0.149143000 \\
7 & -0.834395000 & 3.205039000 & 1.104878000 \\
7 & -6.394353000 & -0.433326000 & -0.112091000 \\
7 & -2.391447000 & -0.641420000 & -0.598348000 \\
7 & -4.462640000 & -1.659798000 & -0.046956000 \\
1 & 5.107783000 & -1.815592000 & -0.590243000 \\
1 & 1.236720000 & -5.124220000 & -0.623493000 \\
6 & 3.062626000 & 0.068581000 & 1.365037000 \\
6 & 3.860486000 & -1.074824000 & 1.000974000 \\
6 & 4.500518000 & -1.007874000 & -0.196760000 \\
1 & 3.929364000 & -1.951623000 & 1.631568000 \\
1 & 1.594305000 & 0.713712000 & 2.597575000 \\
1 & 2.195114000 & -0.838730000 & 2.966652000 \\
7 & 4.393728000 & 0.105304000 & -0.962659000 \\
7 & 2.969849000 & 1.156292000 & 0.615673000 \\
7 & 2.367739000 & 0.055926000 & 2.533426000 \\
6 & 0.252905000 & -2.219740000 & 0.839301000 \\
6 & 0.465893000 & -3.636519000 & 0.721345000 \\
6 & 1.056125000 & -4.075730000 & -0.416758000 \\
1 & 0.121623000 & -4.323509000 & 1.481886000 \\
1 & -0.463402000 & -0.679647000 & 1.978957000 \\
1 & -0.839261000 & -2.298502000 & 2.546329000 \\
7 & 1.451167000 & -3.188837000 & -1.374792000 \\
7 & -3.617982000 & -1.367106000 & -0.125046000 \\
7 & -0.292421000 & -1.696680000 & 1.949550000 \\
\hline & -0.402562000 & 1.920397000 & 1.049432000 \\
\hline & -2.487252000 & -2.842251000 & -0.023085000 \\
\hline
\end{tabular}




$\begin{array}{lrrr}\mathrm{O} & 0.068545000 & 2.645423000 & -0.003738000 \\ \mathrm{~N} & 0.748680000 & -1.428215000 & 0.006708000 \\ \mathrm{~N} & -2.184906000 & 0.511003000 & 0.008218000 \\ \mathrm{~N} & -1.669759000 & -1.656587000 & -0.004823000 \\ \mathrm{~N} & 3.001409000 & -0.909993000 & -0.068230000 \\ \mathrm{C} & -0.816457000 & 0.429054000 & 0.009464000 \\ \mathrm{C} & -0.518684000 & -0.921109000 & -0.000255000 \\ \mathrm{C} & 0.189124000 & 1.438338000 & 0.002792000 \\ \mathrm{C} & 1.679639000 & -0.533365000 & -0.003801000 \\ \mathrm{C} & -2.633837000 & -0.767495000 & -0.000412000 \\ \mathrm{H} & -2.728636000 & 1.357254000 & 0.010864000 \\ \mathrm{H} & -3.686483000 & -0.998141000 & -0.002928000 \\ \mathrm{H} & 3.125961000 & -1.898577000 & 0.084583000 \\ \mathrm{H} & 3.667202000 & -0.333683000 & 0.420531000 \\ \mathrm{~N} & 1.453428000 & 0.823313000 & -0.005641000 \\ \mathrm{H} & 2.232924000 & 1.460577000 & -0.083488000\end{array}$

Guanine dimer B3LYP/6-31++G(d,p)

$\begin{array}{lrrr}\text { O } & 1.186112000 & -1.505788000 & 1.021775000 \\ \text { N } & 3.283929000 & 1.779865000 & -0.417858000 \\ \text { N } & 4.054767000 & -1.734026000 & -0.185194000 \\ \text { N } & 1.407270000 & 2.985544000 & 0.234667000 \\ \text { C } & 3.216520000 & -0.660814000 & 0.030112000 \\ \text { C } & 3.790988000 & 0.527255000 & -0.419746000 \\ \text { C } & 1.907185000 & -0.594192000 & 0.597921000 \\ \mathrm{C} & 2.087984000 & 1.819948000 & 0.123547000 \\ \mathrm{C} & 5.107989000 & -1.212182000 & -0.741960000 \\ \mathrm{H} & 5.989119000 & -1.755214000 & -1.053046000 \\ \mathrm{H} & 1.788313000 & 3.771096000 & -0.266345000 \\ \mathrm{H} & 0.409748000 & 2.952575000 & 0.410014000 \\ \mathrm{~N} & 1.445569000 & 0.724008000 & 0.636382000 \\ \mathrm{H} & 0.506734000 & 0.901822000 & 1.021507000 \\ \mathrm{~N} & 5.007640000 & 0.155132000 & -0.911714000 \\ \mathrm{H} & 5.686681000 & 0.778753000 & -1.319528000 \\ \mathrm{O} & -1.186115000 & 1.505794000 & 1.021768000 \\ \mathrm{~N} & -3.283926000 & -1.779867000 & -0.417855000 \\ \mathrm{~N} & -4.054774000 & 1.734022000 & -0.185194000 \\ \mathrm{~N} & -1.407264000 & -2.985540000 & 0.234671000 \\ \mathrm{C} & -3.216523000 & 0.660814000 & 0.030110000 \\ \mathrm{C} & -3.790988000 & -0.527258000 & -0.419745000 \\ \mathrm{C} & -1.907186000 & 0.594197000 & 0.597915000 \\ \mathrm{C} & -2.087980000 & -1.819946000 & 0.123548000 \\ \mathrm{C} & -5.107995000 & 1.212174000 & -0.741958000 \\ \mathrm{H} & -5.989128000 & 1.755203000 & -1.053043000 \\ \mathrm{H} & -1.788304000 & -3.771094000 & -0.266339000 \\ \mathrm{H} & -0.409741000 & -2.952569000 & 0.410018000 \\ \mathrm{~N} & -1.445565000 & -0.724001000 & 0.636374000 \\ \mathrm{H} & -0.506732000 & -0.901813000 & 1.021509000 \\ \mathrm{~N} & -5.007641000 & -0.155139000 & -0.911713000 \\ \mathrm{H} & -5.686681000 & -0.778764000 & -1.319524000\end{array}$




\begin{tabular}{lrrr}
1 & 4.383541000 & 1.413197000 & 0.104112000 \\
1 & 4.960861000 & 3.701976000 & -0.891331000 \\
7 & 0.471883000 & 1.828731000 & 1.639921000 \\
7 & -1.242291000 & 3.400813000 & 1.649443000 \\
1 & -1.934559000 & 2.696192000 & 1.904039000 \\
1 & -1.562087000 & 4.311661000 & 1.361054000 \\
6 & 0.030299000 & 3.085576000 & 1.284463000 \\
7 & 0.765090000 & 3.973159000 & 0.644839000 \\
6 & 2.029389000 & 3.555037000 & 0.361476000 \\
6 & 2.541609000 & 2.298197000 & 0.709083000 \\
6 & 1.749530000 & 1.328167000 & 1.371431000 \\
7 & 3.834347000 & 2.263537000 & 0.223375000 \\
6 & 4.027064000 & 3.460280000 & -0.402371000 \\
7 & 2.979034000 & 4.271303000 & -0.331470000 \\
1 & -0.189830000 & 1.150153000 & 2.047122000 \\
8 & 2.070524000 & 0.168217000 & 1.695823000 \\
7 & -1.855944000 & -2.479960000 & 1.360356000 \\
1 & -1.468168000 & -3.376354000 & 1.089885000 \\
7 & 0.271539000 & -1.928117000 & 2.129510000 \\
1 & 0.599899000 & -2.685097000 & 1.535422000 \\
1 & 0.945782000 & -1.151323000 & 2.198992000 \\
6 & -0.999163000 & -1.535733000 & 1.901330000 \\
7 & -1.401239000 & -0.309633000 & 2.198147000 \\
6 & -2.697982000 & -0.043496000 & 1.903667000 \\
6 & -3.601634000 & -0.960159000 & 1.358567000 \\
8 & -3.816759000 & -3.127259000 & 0.342102000 \\
6 & -3.190791000 & -2.265413000 & 0.978156000 \\
7 & -4.772443000 & -0.250862000 & 1.153624000 \\
1 & -5.563377000 & -0.554961000 & 0.602184000 \\
6 & -4.526269000 & 1.023545000 & 1.550602000 \\
1 & -5.273627000 & 1.800112000 & 1.476260000 \\
7 & -3.296134000 & 1.191394000 & 2.023017000 \\
1 & 3.517037000 & -4.458677000 & 0.951934000 \\
7 & -1.899225000 & -1.595311000 & -1.850495000 \\
7 & -0.904446000 & -0.728711000 & -1.873517000 \\
1 & 5.914794000 & -3.715448000 & 1.408581000 \\
7 & -2.371029000 & -3.523661000 & -1.451908000 \\
7 & 1.809224000 & -1.294353000 & -1.299553000 \\
7 & 2.437987000 & 0.753183000 & -2.197788000 \\
1 & 1.474654000 & 1.096511000 & -2.133101000 \\
1 & 3.139799000 & 1.479715000 & -2.200556000 \\
6 & 2.789941000 & -0.341251000 & -1.469141000 \\
7 & 4.019158000 & -0.462975000 & -0.996890000 \\
6 & 4.250494000 & -1.618069000 & -0.324635000 \\
6 & 3.289207000 & -2.618152000 & -0.121237000 \\
6 & 1.964897000 & -2.476315000 & -0.591148000 \\
7 & 3.919348000 & -3.588170000 & 0.638291000 \\
\hline & 5.193982000 & -3.141043000 & 0.843355000 \\
\hline & 5.433602000 & -1.967196000 & 0.284677000 \\
\hline & -341587000 & -1.093621000 & -1.619267000 \\
\hline & & & \\
1 & -9387000 & -3.260551000 & -0.414955000 \\
\hline
\end{tabular}




$\begin{array}{llll}6 & -1.272815000 & 0.578469000 & -1.858391000 \\ 6 & -2.588337000 & 1.035488000 & -1.836910000 \\ 8 & -4.897131000 & 0.377650000 & -1.746893000 \\ 6 & -3.690613000 & 0.133828000 & -1.822744000 \\ 7 & -2.491372000 & 2.415912000 & -1.763459000 \\ 1 & -3.263156000 & 3.064946000 & -1.719479000 \\ 6 & -1.163655000 & 2.719228000 & -1.748489000 \\ 1 & -0.789372000 & 3.729698000 & -1.663314000 \\ 7 & -0.398763000 & 1.636240000 & -1.809554000\end{array}$

Thymine monomer B3LYP/6-31++G(d,p)

$\begin{array}{lrrr}\mathrm{O} & 1.389494000 & 1.888441000 & 0.000017000 \\ \mathrm{O} & -2.882991000 & 0.229140000 & 0.000021000 \\ \mathrm{~N} & -0.725792000 & 1.032764000 & -0.000007000 \\ \mathrm{~N} & -1.121526000 & -1.241836000 & -0.000004000 \\ \mathrm{C} & 1.155911000 & -0.489831000 & -0.000008000 \\ \mathrm{C} & 0.673273000 & 0.895196000 & -0.000023000 \\ \mathrm{C} & 2.637845000 & -0.720078000 & 0.000003000 \\ \mathrm{C} & -1.678258000 & 0.028654000 & -0.000013000 \\ \mathrm{H} & -1.084827000 & 1.980701000 & 0.000011000 \\ \mathrm{H} & -1.786321000 & -2.002083000 & 0.000007000 \\ \mathrm{H} & 3.102037000 & -0.259630000 & 0.878067000 \\ \mathrm{H} & 3.102046000 & -0.259646000 & -0.878065000 \\ \mathrm{H} & 2.869001000 & -1.788712000 & 0.000014000 \\ \mathrm{C} & 0.237661000 & -1.483426000 & -0.000004000 \\ \mathrm{H} & 0.518687000 & -2.530854000 & 0.000005000\end{array}$

Thymine dimer B3LYP/6-31++G(d,p)

$\begin{array}{lrrr}\mathrm{O} & -5.330685000 & 1.332094000 & -0.000001000 \\ \mathrm{O} & -0.802628000 & 1.566248000 & 0.000000000 \\ \mathrm{~N} & -3.067497000 & 1.411360000 & 0.000001000 \\ \mathrm{~N} & -1.782783000 & -0.490768000 & 0.000001000 \\ \mathrm{C} & -4.160645000 & -0.732947000 & 0.000000000 \\ \mathrm{C} & -4.287384000 & 0.722688000 & -0.000001000 \\ \mathrm{C} & -5.419903000 & -1.536312000 & 0.000000000 \\ \mathrm{C} & -1.810331000 & 0.869444000 & -0.000001000 \\ \mathrm{H} & -3.118375000 & 2.420808000 & 0.000002000 \\ \mathrm{H} & -0.853100000 & -0.928912000 & 0.000001000 \\ \mathrm{H} & -6.024448000 & -1.297742000 & -0.874708000 \\ \mathrm{H} & -6.024448000 & -1.297740000 & 0.874709000 \\ \mathrm{H} & -5.200956000 & -2.602007000 & 0.000001000 \\ \mathrm{C} & -2.924810000 & -1.254708000 & 0.000000000 \\ \mathrm{H} & -2.752306000 & -2.322178000 & 0.000000000 \\ \mathrm{O} & 5.330690000 & -1.332086000 & 0.000000000 \\ \mathrm{O} & 0.802633000 & -1.566258000 & 0.000001000 \\ \mathrm{~N} & 3.067502000 & -1.411361000 & -0.000001000 \\ \mathrm{~N} & 1.782780000 & 0.490762000 & 0.000001000 \\ \mathrm{C} & 4.160641000 & 0.732950000 & 0.000000000 \\ \mathrm{C} & 4.287386000 & -0.722684000 & -0.000002000 \\ \mathrm{C} & 5.419896000 & 1.536321000 & 0.000000000 \\ \mathrm{C} & 1.810334000 & -0.869450000 & 0.000001000 \\ \mathrm{H} & 3.118384000 & -2.420808000 & -0.000001000 \\ \mathrm{H} & 0.853095000 & 0.928903000 & 0.000000000 \\ \mathrm{H} & 6.024443000 & 1.297752000 & -0.874707000 \\ \mathrm{H} & 6.024441000 & 1.297752000 & 0.874709000\end{array}$




$\begin{array}{llll}\mathrm{H} & 5.200945000 & 2.602015000 & 0.000001000 \\ \mathrm{C} & 2.924804000 & 1.254706000 & 0.000000000 \\ \mathrm{H} & 2.752296000 & 2.322175000 & 0.000000000\end{array}$

$\underline{\text { Thymine cluster B3LYP/6-31++G(d,p) }}$

\begin{tabular}{|c|c|c|c|}
\hline & v & 00 & 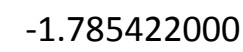 \\
\hline & 3.566919000 & -0.069126000 & -1.838000000 \\
\hline & 2.033844000 & 1.371269000 & -1.754494000 \\
\hline & 4.306333000 & 0.722142000 & -1.861188000 \\
\hline & 6.019167000 & -1.052825000 & -1.920692000 \\
\hline & 5.276678000 & 4.165156000 & 0.684184000 \\
\hline & 4.391655000 & 3.343278000 & 0.900981000 \\
\hline & 4.565104000 & 1.894535000 & 0.945388000 \\
\hline & 3.473195000 & 1.124611000 & 1.182225000 \\
\hline & 5.936514000 & 46000 & 0.717522000 \\
\hline 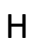 & 32000 & 375000 & 1.227693000 \\
\hline-1 & 5.915470000 & 390000 & 0.753852000 \\
\hline - & 6.333267000 & 1.645872000 & -0.254171000 \\
\hline & 6.642135000 & 1.695052000 & 1.471338000 \\
\hline & 1.540975000 & 334000 & -1.651435000 \\
\hline $\mathrm{N}$ & 0.03 & 0000 & 0000 \\
\hline $\mathrm{N}$ & -0.70 & 7000 & 20000 \\
\hline & 0.365612000 & 3.444973000 & -1.535272000 \\
\hline & 0.809220000 & 129000 & -1.481630000 \\
\hline H & -0.475607000 & 1.584673000 & -1.529494000 \\
\hline 0 & -0.02 & 58000 & 1.650067000 \\
\hline o & -2.97 & 58000 & 1.797283000 \\
\hline V & -1.5 & 8000 & 00000 \\
\hline & -1.209771000 & 97000 & 19000 \\
\hline - & -2.81 & 9000 & 2000 \\
\hline C & -3.89 & 26000 & 13000 \\
\hline C & -5.31 & 2000 & 53000 \\
\hline $\mathrm{H}$ & -0.7 & 9000 & 30000 \\
\hline $\mathrm{H}$ & -5.47 & 35000 & 2.678659000 \\
\hline H & -5.518906000 & 6000 & 25000 \\
\hline $\mathrm{O}$ & 0.822569000 & 3.477109000 & 1.443849000 \\
\hline N & 2.218949000 & 130000 & 1.367248000 \\
\hline N & 3.07 & 5000 & 13000 \\
\hline C & 1.96 & 3000 & 6000 \\
\hline $\mathrm{H}$ & 1.402273000 & 5000 & 8000 \\
\hline $\mathrm{H}$ & 2.922680000 & 4.782319000 & 1.106233000 \\
\hline 0 & -2.904848000 & 2.061308000 & -1.274521000 \\
\hline C & -2.046808000 & 34000 & -1.335976000 \\
\hline C & -2.32 & 00 & 3000 \\
\hline C & -1.264961000 & 5.221601000 & -1.331887000 \\
\hline C & -3.738421000 & 4.831248000 & -1.100356000 \\
\hline $\mathrm{H}$ & -1.377399000 & 6.297742000 & -1.270801000 \\
\hline $\mathrm{H}$ & -3.80 & 1000 & -1.166252000 \\
\hline $\mathrm{H}$ & -4.11 & 736000 & -0.122467000 \\
\hline $\mathrm{H}$ & -4.380646000 & 4.388451000 & -1.867940000 \\
\hline $\mathrm{O}$ & 0.028947000 & -0.087270000 & -1.650517000 \\
\hline $\mathrm{O}$ & 2.975066000 & -3.580846000 & -1.796231000 \\
\hline $\mathrm{N}$ & 1.518796000 & -1.826064000 & -1.745781000 \\
\hline C & 1.209676000 & -0.498903000 & -1.721574000 \\
\hline C & 2.817438000 & -2.364446000 & -1.794893000 \\
\hline$C$ & 3.898388000 & -1.380704000 & -1.83963600 \\
\hline
\end{tabular}




$\begin{array}{llll}\mathrm{C} & 5.310360000 & -1.881381000 & -1.844455000 \\ \mathrm{H} & 0.728500000 & -2.488654000 & -1.688690000 \\ \mathrm{H} & 5.479174000 & -2.571110000 & -2.677234000 \\ \mathrm{H} & 5.518547000 & -2.435761000 & -0.922944000 \\ \mathrm{O} & 2.905074000 & -2.060826000 & 1.275260000 \\ \mathrm{C} & 2.047171000 & -2.943493000 & 1.336661000 \\ \mathrm{C} & 2.321119000 & -4.377147000 & 1.270703000 \\ \mathrm{C} & 1.265693000 & -5.221385000 & 1.332480000 \\ \mathrm{C} & 3.739221000 & -4.830630000 & 1.102150000 \\ \mathrm{H} & 1.378332000 & -6.297511000 & 1.271503000 \\ \mathrm{H} & 3.810275000 & -5.919575000 & 1.168301000 \\ \mathrm{H} & 4.113472000 & -4.514134000 & 0.124435000 \\ \mathrm{H} & 4.380964000 & -4.387506000 & 1.869954000 \\ \mathrm{O} & -0.822917000 & -3.477168000 & -1.444635000 \\ \mathrm{~N} & -2.219137000 & -1.657074000 & -1.367513000 \\ \mathrm{~N} & -3.075027000 & -3.781874000 & -1.142067000 \\ \mathrm{C} & -1.960327000 & -2.998639000 & -1.325083000 \\ \mathrm{H} & -1.402381000 & -1.042793000 & -1.496210000 \\ \mathrm{H} & -2.923182000 & -4.782324000 & -1.107951000 \\ \mathrm{~N} & -2.259660000 & -0.364477000 & 1.785422000 \\ \mathrm{C} & -3.566940000 & 0.068775000 & 1.838338000 \\ \mathrm{H} & -2.033766000 & -1.371473000 & 1.754215000 \\ \mathrm{H} & -4.306282000 & -0.722560000 & 1.861494000 \\ \mathrm{H} & -6.019254000 & 1.052233000 & 1.921539000 \\ \mathrm{O} & -1.540679000 & -3.067513000 & 1.651036000 \\ \mathrm{~N} & -0.033006000 & -4.774527000 & 1.475204000 \\ \mathrm{~N} & 0.701596000 & -2.591290000 & 1.474072000 \\ \mathrm{C} & -0.365239000 & -3.444995000 & 1.535163000 \\ \mathrm{H} & -0.808524000 & -5.421221000 & 1.481353000 \\ \mathrm{H} & 0.475665000 & -1.584565000 & 1.529363000 \\ \mathrm{O} & -5.277209000 & -4.165155000 & -0.686079000 \\ \mathrm{C} & -4.392090000 & -3.343259000 & -0.902429000 \\ \mathrm{C} & -4.565402000 & -1.894482000 & -0.946197000 \\ \mathrm{C} & -3.473378000 & -1.124543000 & -1.182457000 \\ \mathrm{C} & -5.936824000 & -1.330491000 & -0.718407000 \\ \mathrm{H} & -3.508014000 & -0.040782000 & -1.227405000 \\ \mathrm{H} & -5.915683000 & -0.238319000 & -0.754193000 \\ \mathrm{H} & -6.333878000 & -1.646266000 & 0.253016000 \\ \mathrm{H} & -6.642261000 & -1.694570000 & -1.472602000\end{array}$




\section{Acknowledgement}

This work was supported by the National Science Center Poland, Grant 2017/27/B/ST4/00948.

This work was supported by the National Science Center Poland, Grant 2016/21/B/ST4/02102.

Calculations have been carried out in Wroclaw Centre for Networking and Supercomputing (http:/www.wcss.pl), under Grant no. 163. 